\title{
Rogue waves and rational solutions of the Hirota equation
}

\author{
Adrian Ankiewicz, ${ }^{1}$ J. M. Soto-Crespo, ${ }^{2}$ and Nail Akhmediev ${ }^{1}$ \\ ${ }^{1}$ Optical Sciences Group, Research School of Physics and Engineering, Institute of Advanced Studies, \\ The Australian National University, Canberra, Australian Capital Territory 0200, Australia \\ ${ }^{2}$ Instituto de Óptica, CSIC, Serrano 121, 28006 Madrid, Spain \\ (Received 12 November 2009; published 15 April 2010)
}

\begin{abstract}
The Hirota equation is a modified nonlinear Schrödinger equation (NLSE) that takes into account higherorder dispersion and time-delay corrections to the cubic nonlinearity. In describing wave propagation in the ocean and optical fibers, it can be viewed as an approximation which is more accurate than the NLSE. We have modified the Darboux transformation technique to show how to construct the hierarchy of rational solutions of the Hirota equation. We present explicit forms for the two lower-order solutions. Each one is a regular (nonsingular) rational solution with a single maximum that can describe a rogue wave in this model. Numerical simulations reveal the appearance of these solutions in a chaotic field generated from a perturbed continuous wave solution.
\end{abstract}

DOI: 10.1103/PhysRevE.81.046602

PACS number(s): 42.65.Tg, 47.20.Ky, 47.35.Fg

\section{INTRODUCTION}

As pointed out by many researchers, rogue waves can appear in the ocean and in optical fibers due to modulation instability (MI) [1-3]. The latter is widely known as the Benjamin-Feir [4] or Bespalov-Talanov [5] instability. Specifically, a periodic perturbation on the top of a continuous wave exponentially increases its amplitude due to the above instability. Waves in a continuous but limited frequency range are involved in this dynamics. Modulation instability is known for a number of nonlinear equations, with the most important one being the nonlinear Schrödinger equation (NLSE)

$$
i \frac{\partial \psi}{\partial x}+\frac{1}{2} \frac{\partial^{2} \psi}{\partial t^{2}}+|\psi|^{2} \psi=0
$$

where $x$ is the propagation variable and $t$ is the retarded time variable in a moving frame while $\psi$ is the envelope of the wave field. It is worthwhile to mention here that the NLSE was first derived by Lev Ostrovsky [6].

For each frequency, the long term dynamics of MI is closely related to the so called Fermi-Pasta-Ulam recurrence [7]. Namely, during the initial stages of the development, each particular frequency mode in a modulation instability phenomenon receives its energy from the central mode, along with the higher harmonics and then returns all the energy back to the central mode [8]. For the particular case of the NLSE, this process can be described by an exact solution that has been referred to as the "Akhmediev Breather" $(\mathrm{AB})$ in a number of earlier works on ocean waves [9-13]. This solution describes the appearance of a periodic sequence of maxima on the top of a continuous wave and their subsequent disappearance on further evolution [14].

A particular case of modulation instability is the one with a zero frequency (or infinite period) sideband. In this case, the periodic sequence of maxima is reduced to just a single peak which "appears from nowhere and disappears without a trace" [15]. The latter is also described by an exact solution of the NLSE, which has a simple rational representation. The lowest-order solution of this class is known as the Peregrine soliton [16] and can be obtained as a limiting case of an $\mathrm{AB}$ with zero frequency of modulation [17]. There is a hierarchy of rational solutions with progressively increasing amplitude [18]. Each of them can be thought of as a rogue wave. Moreover, the high amplitude waves described by the higher-order rational solutions appear naturally in the chaotic wave field as a nonlinear superposition of two or more ABs initiated by modulation instability with nonzero frequency sidebands $[19,20]$. Thus, it is essential to know the structure of rational solutions in order to understand rogue waves in general.

However, the phenomenon of ocean waves is significantly more complicated than the one modeled by the simple NLSE. Even in the one-dimensional case, higher-order terms that take into account third-order dispersion, self-steepening and other nonlinear effects have to be added to this equation $[21,22]$. Thus, a question arises: do the rational solutions, which are prototypes of rogue waves, exist for these more complicated equations? Of course, it is impossible to answer this question in one step. We have partially analyzed the problem in one of our previous works [23]. In the present publication, we make one more step forward in an attempt to understand this issue. Namely, we take an equation which is a further modification of the NLSE with the addition of terms that are responsible for third-order dispersion and a time-delay correction to the cubic nonlinearity. In particular, we take the coefficients in such a way that we arrive at an integrable model. This model is well-known as the Hirota equation (HE). In dimensionless form it is given by

$$
i \frac{\partial \psi}{\partial x}+\frac{1}{2} \frac{\partial^{2} \psi}{\partial t^{2}}+|\psi|^{2} \psi-i \alpha_{3} \frac{\partial^{3} \psi}{\partial t^{3}}-i 6 \alpha_{3}|\psi|^{2} \frac{\partial \psi}{\partial t}=0,
$$

where the variables $x$ and $t$ have the same physical meaning as in Eq. (1). The two terms in Eq. (2) that enter with a real coefficient $\alpha_{3}$ are responsible for the third-order dispersion and a time-delay correction to the cubic term, respectively. It is easy to see that if we set $\alpha_{3}=0$, then we obtain the NLSE (1). Of course, it may be too much to expect that the results could somehow be directly applied in the water wave context, especially taking into account the fact that the second 
dimension is unavoidable in the vast space of the world's oceans. On the other hand, in optics, it is common to create fibers with the desired properties. We cannot exclude that in future it may become possible to produce a fiber where propagation is described by the Hirota equation.

A number of higher-order equations related to water waves have been considered by Osborne in [24]. They allow approximate integration, unlike the $\mathrm{HE}$ which can be integrated completely.

The HE was introduced in [25] and studied in a number of papers [26-28]. It is an integrable equation which can be considered as a combination of the NLSE (where $\psi$ is complex) and the modified Korteweg de Vries (KdV) equation

$$
\frac{\partial \psi}{\partial x}+\frac{\partial^{3} \psi}{\partial t^{3}}+6 \psi^{2} \frac{\partial \psi}{\partial t}=0
$$

where $\psi$ is real. Indeed, if we eliminate the second order derivative and cubic nonlinear terms in Eq. (2), consider $\psi$ as real and take $\alpha_{3}=-1$, then we arrive at Eq. (3). The $\mathrm{mKdV}$ equation is also known to be a completely integrable equation that has all the basic features of integrable models. Although combining two completely integrable equations into a single one does not automatically provide integrability, this is however the case in this particular instance. On the other hand, despite producing the integrability, this combination does not automatically provide us with solutions to the resulting integrable equation. Generally, we cannot use the solutions of either the NLSE or the mKdV and modify them in a simple way to produce a solution of the HE. This approach may work to some extent for the lowest-order solutions such as single solitons or first-order ABs but for more complicated solutions we have to derive them using more sophisticated techniques. From simple analysis, we know that the main effect of the mKdV terms added to the NLSE is to introduce a "tilt" to the original NLSE solutions [27]. We consider this assumption in more detail below for the case of rational solutions.

First, let us show that the modified KdV equation by itself does not have a "rogue wave" solution that could be similar to the Peregrine soliton in the case of the NLSE. In order to show this we start with a breather solution of the mKdV Eq. [29]

$$
\psi(x, t)=-4 q \frac{a}{b} \operatorname{sech}(\theta)
$$

where $\quad a=\cos (\phi)-\frac{q}{p} \sin (\phi) \tanh (\theta), \quad$ while $\quad b=1$ $+\left[\frac{q}{p} \sin (\phi) \operatorname{sech}(\theta)\right]^{2}, \quad \theta=2 q t+8 q\left(3 p^{2}-q^{2}\right) x, \quad$ and $\quad \phi=2 p t$ $+8 p\left(p^{2}-3 q^{2}\right) x$. The solution has two free parameters $p$ and $q$. It is shown in Fig. 1 for the case $p=0.45$, and $q=0.5$. The period of the "hills" in the solution is defined by the parameter $q$. When $q \rightarrow 0$, the period becomes infinitely large. Taking the approach that the rogue wave is the limit of the breather solution when the period increases to infinity, we can check that the amplitude of the hills actually decreases to zero $(\psi \rightarrow 0)$ when $q \rightarrow 0$. Thus, for the limit in which we are interested, the solution degenerates to the trivial one. Physically, this is a mere consequence of the fact that the $\mathrm{mKdV}$ breather sits on a zero background for any values of the two

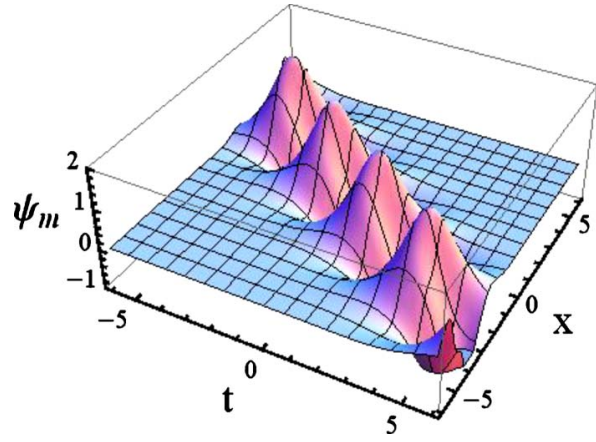

FIG. 1. (Color online) Breather solution of the modified KdV Eq. (4) for $p=0.45$ and $q=0.5$.

parameters $p$ and $q$. Thus, there is not a single-maximum rogue wave described by the $\mathrm{mKdV}$ equation. We do not consider here the soliton solutions that do not belong to the class of solutions that "appear from nowhere" [15].

The single AB solution of the Hirota Eq. (2) which is seeded by modulation instability can be derived using a special ansatz similar to that used in Chapter 3 [specifically Eq. (3.1)] of [30]. It was obtained previously in [27]. It is given by

$$
\psi=\left[\frac{2\left(1-2 a_{1}\right) \cosh (\beta x)+i \beta \sinh (\beta x)}{\cosh (\beta x)-\sqrt{2 a_{1}} \cos [s(t+v x)]}-1\right] e^{i x},
$$

where $s=2 \sqrt{1-2 a_{1}}$, and $\beta=\sqrt{2 a_{1}} s$ while $a_{1}$ is an arbitrary number in the range $0<a_{1}<0.5$ and $v=2 \alpha_{3}\left(1+4 a_{1}\right)$. Equation (5) is an exact solution of Eq. (2) with one free parameter, $a_{1}$, which defines the period of the solution in the $t$ direction. This solution is shown in Fig. 2 for $a_{1}=0.2$ and $\alpha_{3}=0.2$.

Note that $\psi(x, t)$ is not a function of $(t+v x)$ only. Thus, $v$ is not the "velocity" of the breather, although it introduces the "tilt" to the "hills" of the solution. The maxima of the HE ABs are located on a line which is parallel to the $x$-axis. This differentiates it from the AB of the NLSE with velocity [20], where the line of maxima is located at an angle to the $x$ axis.

We also note here that rational solutions are limiting cases of either solutions describing solitons on a background or ABs. $N$-soliton solutions are known for the NLSE and HE. However, solitons on a background are a different matter because the boundary conditions are changed. Moreover, pe-

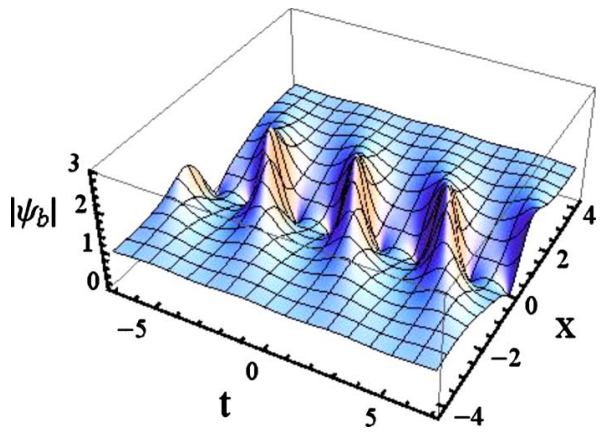

FIG. 2. (Color online) First-order Hirota breather [Eq. (5)] for $a_{1}=0.2$ and $\alpha_{3}=0.2$. 
riodic solutions such as $\mathrm{ABs}$ have eigenvalues that are different from those for solitons. Even if the solutions are found, taking the limit in each case is tricky and has to be done carefully. This may be relatively simple for the lowestorder solutions, but for the higher-order ones, it becomes an increasingly nontrivial exercise. This is the reason why higher-order rational solutions have remained unknown until now. Thus, developing alternative techniques is one of the ways to overcome this difficulty.

\section{MODULATION INSTABILITY AND ABS OF THE HIROTA EQUATION}

We can analyze the development of modulation instability of a continuous wave (CW) $[14,27,30]$ by setting $\psi$ $=u(x, t) \exp (i x)$ where we fixed the wave number of the $\mathrm{CW}$ to be 1 . We take the solution for $u$ as a constant with a small periodic perturbation

$$
u(x, t)=1+[a(x)+i b(x)] \cos [\kappa(t+v x)],
$$

where $\kappa$ is the period of the perturbation and $a(x)$ and $b(x)$ are small real values that represent the complex amplitude of the periodic perturbation. Linearizing $u$ around 1 shows that the parameter $v$ is given by [27]

$$
v=\alpha_{3}\left(6-\kappa^{2}\right) .
$$

Further, in the linear approximation we arrive at the following set of two coupled equations:

$$
\begin{gathered}
\left(\kappa^{2}-4\right) a(x)+2 b^{\prime}(x)=0, \\
\kappa^{2} b(x)=2 a^{\prime}(x) .
\end{gathered}
$$

Restricting ourselves to exponentially growing solutions $\sim e^{\delta x}$ we obtain

$$
\begin{gathered}
a(x)=\nu \exp (\delta x), \\
b(x)=2 \nu \frac{\delta}{\kappa^{2}} \exp (\delta x),
\end{gathered}
$$

where $\nu=a(0)$ is a small initial amplitude. The instability growth rate is given by

$$
\delta=\kappa \sqrt{1-\frac{\kappa^{2}}{4}} .
$$

This expression for the growth rate is the same as for the NLSE [27]. Consequently, the initial modulation grows exponentially if its frequency is in the range $0<\kappa<2$. The maximum value of the growth rate is 1 , and this occurs when $\kappa=\sqrt{2}$.

Based on this analysis, we can rewrite the solution of Eq. (5) in terms of $\kappa$ by setting $a_{1}=\left(4-\kappa^{2}\right) / 8$. Then $s=\kappa, \beta=\delta$, and $v=\alpha_{3}\left(6-\kappa^{2}\right)$ in agreement with Eq. (6) above. A simple transformation to new variables leads to

$$
\psi=\left[\kappa \frac{(\kappa p / 2) \cosh (\delta x)+i \sinh (\delta x)}{p \cosh (\delta x)-\cos [\kappa(t+v x)]}-1\right] e^{i x},
$$

where $p=\kappa / \delta$. The frequency $\kappa$ is a free parameter of this solution. Equation (8) describes completely the evolution of

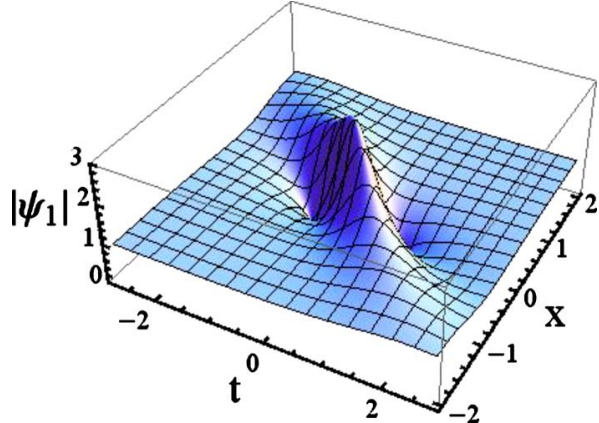

FIG. 3. (Color online) First-order rational solution of Hirota equation [Eq. (9)] for $\alpha_{3}=0.2$. The arbitrary real parameter $\alpha_{3}$ is responsible for the skew angle of the "ridge" of this solution relative to the axes.

the nonlinear wave field for every $\kappa$ within the instability range. After certain propagation distance, this solution returns back to the continuous wave solution. This happens with the negative exponential $\sim e^{-\delta x}$ which was ignored in the above approximation. Just like the AB of the NLSE, the ABs (8) can be considered as the exact solution of the FermiPasta-Ulam recurrence problem $[8,31]$ for the Hirota equation. Namely, initially all the energy is contained in a central frequency mode which is a continuous wave. Upon evolution, the energy is distributed between the modulationally unstable sidebands and their harmonics. This happens until the maximum of modulation is reached. After reaching the highest point of modulation, all the energy returns back to the original mode, i.e., to the continuous wave.

The solution (8) is valid in the whole frequency interval $0<\kappa<2$ including the points 0 and 2 . At these border points we have to consider special limits. Taking the limit $\kappa \rightarrow 0$, we have $a_{1} \rightarrow 1 / 2$. Applying the L'Hôpital's rule either to Eq. (5) or to Eq. (8), we obtain

$$
\psi_{1}=-\left[1-4 \frac{1+2 i x}{D_{1}}\right] e^{i x},
$$

where

$$
D_{1}=1+4\left(t+6 \alpha_{3} x\right)^{2}+4 x^{2} .
$$

So here we have

$$
v=6 \alpha_{3} .
$$

Thus, this is the simplest form of the lowest-order rational solution of the Hirota equation. It is shown in Fig. 3. The solution has the same form as the first-order rational solution of the NLSE [15], apart from the fact that $t$ is replaced with $t+v x$. Consequently, the "ridge" of the solution is tilted to the $t$ axis. This can be attributed to the "convective behavior" of systems with higher-order dispersion [32].

The existence of solutions localized in two directions is a remarkable feature of PDEs like the NLSE. Their existence for the Hirota equation is a further confirmation that such solutions play a very special role as prototypes of rogue waves. Our earlier studies revealed that the Hirota equation is not the only extension of the NLSE which has doubly localized structures [23]. However, higher-order solutions of 
this type can only be constructed for integrable equations. In this sense, the Hirota equation is a model that allows us to construct them using existing techniques.

\section{MODIFIED DARBOUX TRANSFORMATION SCHEME}

The "Darboux transformation" (DT) is a step by step technique of constructing composite solutions out of simple ones [33]. In the case of the NLSE, the DT can be used to construct multisoliton solutions out of the trivial zero solution or multi-AB solutions out of simple CW solution [19]. Solutions of either class consist of a corresponding "hierarchy" of solutions depending on the number of solitons or ABs involved in each.

DT cannot be directly used to obtain the hierarchy of rational solutions. It has to be modified to make it applicable to this special class of solutions. Usually, rational solutions can be derived from the solutions of another class taking special limits. For example, the lowest-order rational solution (9) may be obtained either as a limiting case of a $\mathrm{Ma}$ soliton or an AB [19]. Similarly, higher-order solutions can be calculated as the limiting cases of $N$ soliton or $N$-AB solutions. Below, we modify the DT technique in such a way that these limits are taken in the intermediate calculations rather than applied to the solutions themselves. This modification has allowed us to construct the hierarchy of rational solutions directly.

As before [19] we start with the linear set of equations representing the integrable nonlinear equation. For the case of the Hirota equation, it can be written as

$$
\begin{gathered}
\mathbf{R}_{t}=i \mathbf{J R}+\mathbf{U R}, \\
\mathbf{R}_{x}=-\mathbf{J R}+i \mathbf{U R}+\frac{1}{2} \mathbf{V R}+\alpha_{3} \mathbf{M R},
\end{gathered}
$$

where

$$
\mathbf{R}=\left[\begin{array}{l}
r \\
s
\end{array}\right],
$$

with two linear complex functions $r=r(x, t)$ and $s=s(x, t)$ and where $\mathbf{U}, \mathbf{J}$ and $\mathbf{M}$ are the following square matrices:

$$
\begin{gathered}
\mathbf{U}=\left[\begin{array}{cc}
0 & i \psi^{*} \\
i \psi & 0
\end{array}\right], \mathbf{J}=\left[\begin{array}{cc}
i & 0 \\
0 & -i
\end{array}\right], \\
\mathbf{V}=\left[\begin{array}{cc}
-i|\psi|^{2} & \psi_{t}^{*} \\
-\psi_{t} & i \mid \psi^{2}
\end{array}\right], \\
\mathbf{M}=\left[\begin{array}{cc}
-a_{1} & i a_{3} \\
-i a_{2} & a_{1}
\end{array}\right],
\end{gathered}
$$

where

$$
\begin{aligned}
& a_{1}=4+2|\psi|^{2}-\psi \psi_{t}^{*}+\psi^{*} \psi_{t}, \\
& a_{2}=4 \psi+2 \psi_{t}+\psi_{t t}+2 \psi|\psi|^{2},
\end{aligned}
$$

and

$$
a_{3}=4 \psi^{*}-2 \psi_{t}^{*}+\psi_{t t}^{*}+2 \psi^{*}|\psi|^{2} .
$$

Thus, the linear functions $r$ and $s$ depend on which solution $\psi$ we use in the matrices.

The second equation in Eq. (11) can be written in shorter form if we combine several matrices into a single one

$$
\mathbf{R}_{x}=\left[\begin{array}{cc}
w_{11} & w_{12} \\
w_{21} & -w_{11}
\end{array}\right] \mathbf{R},
$$

where

$$
\begin{gathered}
w_{11}=-i-\frac{i}{2}|\psi|^{2}-\alpha_{3}\left(a_{1}\right), \\
w_{12}=-\psi^{*}+\frac{1}{2} \psi_{t}^{*}+i \alpha_{3}\left(a_{3}\right),
\end{gathered}
$$

and

$$
w_{21}=-\psi-\frac{1}{2} \psi_{t}+i \alpha_{3}\left(a_{2}\right) .
$$

Indeed, it is easy to check that the condition of compatibility

$$
\mathbf{R}_{t x}=\mathbf{R}_{x t},
$$

of the linear equations leads directly to the Hirota equation. It is easy to check that, when $\alpha_{3}=0$, the above set of equations is reduced to the linear set presented in [19]. The eigenvalue in Eq. (11) and the following equations has been specified in order to deal with rational solutions. Namely, $\lambda$ $=i$.

Once we solve the above equations for a given (seeding) solution of the Hirota equation $\psi_{j}$, we can find the next level solution using a simple transformation, namely,

$$
\psi_{j}=\psi_{j-1}-\frac{4 i s_{j} r_{j}^{*}}{\left|r_{j}\right|^{2}+\left|s_{j}\right|^{2}} .
$$

where $\psi_{j-1}$ is the solution of the previous step while $\psi_{j}$ is the solution of the next step. We start with a simple "seeding solution" in the form

$$
\psi_{0}=e^{i x} .
$$

This allows us to solve the equations for $r$ and $s$ with ease. Substituting these solutions into the Eq. (17) we obtain the next (first-)order solution of the Hirota equation.

Namely, solving the linear equations, we find

$$
\begin{aligned}
& r_{1}=\frac{1}{\sqrt{2}} \exp \left(-\frac{i x}{2}\right)\left[2\left(t+6 \alpha_{3} x\right)+2 i x-1\right], \\
& s_{1}=\frac{1}{i \sqrt{2}} \exp \left(\frac{i x}{2}\right)\left[2\left(t+6 \alpha_{3} x\right)+2 i x+1\right] .
\end{aligned}
$$

These expressions lead directly to Eq. (9) above, and one can easily verify that it satisfies the Hirota equation. The amplitude profile of this solution is shown in Fig. 3. It is easy to see from this figure its similarity to the rational solution of the NLSE shown in Fig. 5 of [19] except for the finite tilt with respect to the axes. The tilt is related to the finite value 
of $\alpha_{3}$ and is zero when $\alpha_{3}=0$. An interesting observation is that on the central line where $x=0$ the shape is independent of $\alpha_{3}$. This means that the additional terms in the Hirota equation do not have any effect on the rogue wave profile at $x=0$ where the profile has its maximum. Indeed, this profile is the same as that in the NLSE case. Consequently, the overall maximum value of the amplitude profile is $\psi_{1}(0,0)$ $=3$ for any value of $\alpha_{3}$.

\section{SECOND-ORDER RATIONAL SOLUTION}

Linear functions corresponding to the next-higher-order solution of the HE are also complex. We can write them explicitly in the form

$$
\begin{aligned}
& r_{2}=r_{2 r}+i r_{2 i}, \\
& s_{2}=s_{2 r}+i s_{2 i} .
\end{aligned}
$$

Finding these functions using the standard algebraic relations of the DT is not possible as those relations do not produce new solutions when the two eigenvalues involved in the solution coincide. This is exactly our case. Thus, we do not have any other choice but to solve the Eq. (11) once again with the matrices that contain the HE solution $\psi_{j}$ of first order. The linear functions found this way can be written as

$$
\begin{aligned}
r_{2 r}= & m \exp \left(\frac{-i x}{2}\right)\left[-a(x,-t)+\alpha_{3} x c(x,-t)-\alpha_{3}^{2} d(x,-t)\right. \\
& +\alpha_{3}^{3} x b_{1}(x,-t)-\alpha_{3}^{4} x^{2} b_{2}(x,-t)+\alpha_{3}^{5} x^{3} b_{3}(x,-t) \\
& \left.-\alpha_{3}^{6} x^{4} b_{4}\right] / D_{1}, \\
r_{2 i}= & m \exp \left(\frac{-i x}{2}\right)\left[x k(x,-t)-\alpha_{3} p(x, t)+\alpha_{3}^{2} x g(x,-t)\right. \\
& \left.-\alpha_{3}^{3} x^{2} f_{1}(x,-t)+\alpha_{3}^{4} x^{3} f_{2}(x,-t)-\alpha_{3}^{5} x^{4} f_{3}\right] / D_{1}, \\
s_{2 r}= & m \exp \left(\frac{i x}{2}\right)\left[x k(x, t)+\alpha_{3} p(x, t)+\alpha_{3}^{2} x g(x, t)+\alpha_{3}^{3} x^{2} f_{1}(x, t)\right. \\
+ & \left.\alpha_{3}^{4} x^{3} f_{2}(x, t)+\alpha_{3}^{5} x^{4} f_{3}\right] / D_{1}, \\
s_{2 i}= & m \exp \left(\frac{i x}{2}\right)\left[a(x, t)+\alpha_{3} x c(x, t)+\alpha_{3}^{2} d(x, t)+\alpha_{3}^{3} x b_{1}(x, t)\right. \\
& \left.+\alpha_{3}^{4} x^{2} b_{2}(x, t)+\alpha_{3}^{5} x^{3} b_{3}(x, t)+\alpha_{3}^{6} x^{4} b_{4}(x, t)\right] / D_{1},
\end{aligned}
$$

where $m$ is an arbitrary real constant reflecting the fact that linear functions can be of any amplitude and the polynomials $q, a, k, c, p, d, g, b$, and $f$ are defined below.

For convenience, we introduce $q(t)$ as follows:

$$
q(t)=3+12 t+16 t^{3}+16 t^{4} .
$$

The polynomials $q, a, k, c, p, d$, and $g$ are then given by

$$
\begin{gathered}
a(x, t)=-q(t)-8 x^{2}\left(3-6 t+2 x^{2}\right), \\
k(x, t)=-8\left(4 t^{3}+4 x^{2} t-3 t+4 x^{2}+3\right), \\
c(x, t)=24\left(-8 t^{3}-12 t^{2}+8 x^{2} t+2 t-4 x^{2}-1\right),
\end{gathered}
$$

$$
\begin{gathered}
p(x, t)=-6\left[q(t)+16 x^{2}\left(-1+3 t+6 t^{2}+x^{2}\right)\right], \\
d(x, t)=6\left[13 q(t)-8 x^{2}\left(9-42 t+2 x^{2}\right)\right], \\
g(x, t)=48\left(4 t^{3}-36 t^{2}-20 x^{2} t-15 t+16 x^{2}+18\right) .
\end{gathered}
$$

Furthermore, the ' $b$ ' polynomials are given by

$$
\begin{gathered}
b_{1}(x, t)=144\left[13\left(7-8 t+12 t^{2}+16 t^{3}\right)+12(11+4 t) x^{2}\right], \\
b_{2}(x, t)=3456\left(-26+39 t+78 t^{2}+6 x^{2}\right), \\
b_{3}(x, t)=269568(1+4 t), \\
b_{4}=1617408 .
\end{gathered}
$$

And finally the ' $f$ ' set of polynomials is given by

$$
\begin{gathered}
f_{1}(x, t)=288\left(37-36 t+84 t^{2}+28 x^{2}\right), \\
f_{2}(x, t)=20736(9 t-1), \\
f_{3}=414720 .
\end{gathered}
$$

Linear functions given by the above equations allow us to find the complete second-order solution according to Eq. (17).

Although the coefficient $m$ is arbitrary, for convenience of further calculations, we relate it to $\alpha_{3}$ through the equation

$$
2 m^{2}\left(1-120 \alpha_{3}^{2}+6084 \alpha_{3}^{4}\right)=1 .
$$

Defining it this way saves us from writing excessively complicated formulas for $G_{2}, H_{2}$, and $D_{2}$ in the solution below. With this simplification, we can now write the exact secondorder rational solution of the Hirota equation explicitly

$$
\psi_{2}(x, t)=e^{i x}\left(1+\frac{G_{2}+i x H_{2}}{D_{2}}\right),
$$

where

$$
\begin{aligned}
G_{2} / 12= & -16 t^{4}-384 \alpha_{3} x t^{3}-24\left[4\left(36 \alpha_{3}^{2}+1\right) x^{2}+1\right] t^{2} \\
& -96 \alpha_{3} x\left[12\left(12 \alpha_{3}^{2}+1\right) x^{2}+7\right] t-16\left(1296 \alpha_{3}^{4}+216 \alpha_{3}^{2}\right. \\
+ & 5) x^{4}-72\left(44 \alpha_{3}^{2}+1\right) x^{2}+3, \\
H_{2} / 24= & -16 t^{4}-384 \alpha_{3} x t^{3}-8\left[\left(432 \alpha_{3}^{2}+4\right) x^{2}-3\right] t^{2} \\
& -96 \alpha_{3} x t\left[4\left(36 \alpha_{3}^{2}+1\right) x^{2}+1\right]-16\left(36 \alpha_{3}^{2}+1\right)^{2} x^{4} \\
& -8\left(180 \alpha_{3}^{2}+1\right) x^{2}+15,
\end{aligned}
$$

and

$$
\begin{aligned}
D_{2}= & 64 t^{6}+2304 \alpha_{3} x t^{5}-432\left(624 \alpha_{3}^{4}-40 \alpha_{3}^{2}-1\right) x^{4} \\
& +36\left(556 \alpha_{3}^{2}+11\right) x^{2}+9+64\left(36 \alpha_{3}^{2}+1\right)^{3} x^{6} \\
& +384 \alpha_{3} t^{3}\left(12\left(60 \alpha_{3}^{2}+1\right) x^{2}-1\right) x+48 t^{4}\left(\left(720 \alpha_{3}^{2}+4\right) x^{2}\right. \\
& +1)+12\left(16\left(6480 \alpha_{3}^{4}+216 \alpha_{3}^{2}+1\right) x^{4}-24\left(60 \alpha_{3}^{2}+1\right) x^{2}\right. \\
& +9) t^{2}+144 \alpha_{3} x\left(16\left(36 \alpha_{3}^{2}+1\right)^{2} x^{4}+\left(8-864 \alpha_{3}^{2}\right) x^{2}+17\right) t .
\end{aligned}
$$




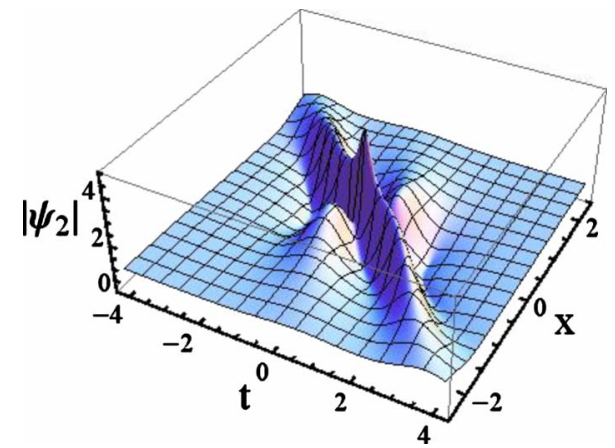

FIG. 4. (Color online) Second-order Hirota rational soliton [Eq. (26)] for $\alpha_{3}=0.17$.

Indeed, the function (26) exactly satisfies the Hirota equation. This can be checked using modern tools for analytic calculations such as "Mathematica" or equivalent, and also numerically. The amplitude profile of this solution is shown in Fig. 4 while the contour plot of the same function is in Fig. 5.

Of course, when $\alpha_{3}=0$, the solution reduces to the NLSE second-order rational solution [19]. On the central line $x=0$, where the solution reaches its maximum, we have

$$
G_{2}(0, t)=12\left(3-24 t^{2}-16 t^{4}\right)
$$

and

$$
D_{2}(0, t)=9+108 t^{2}+48 t^{4}+64 t^{6},
$$

so the wave profile is then independent of $\alpha_{3}$. Remarkably, the function $\psi_{2}(0, t)$ is the same as for the solution of the NLSE. The absolute maximum value of the profile occurs at $(x, t)=(0,0)$ and equals to 5 for any $\alpha_{3}$.

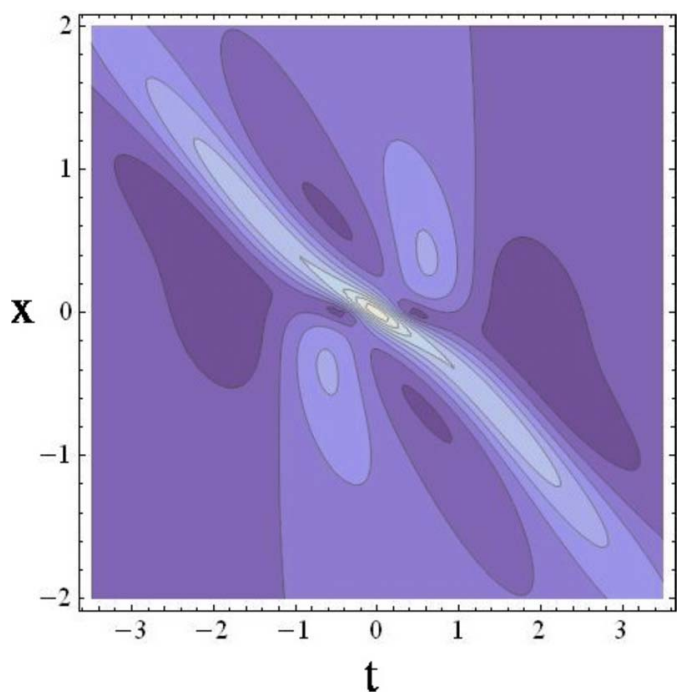

FIG. 5. (Color online) Contour plot [(dimensionless) $t$ horizontal and $x$ vertical] of the second-order Hirota rational soliton [Eq. (26)] for $\alpha_{3}=0.17$.

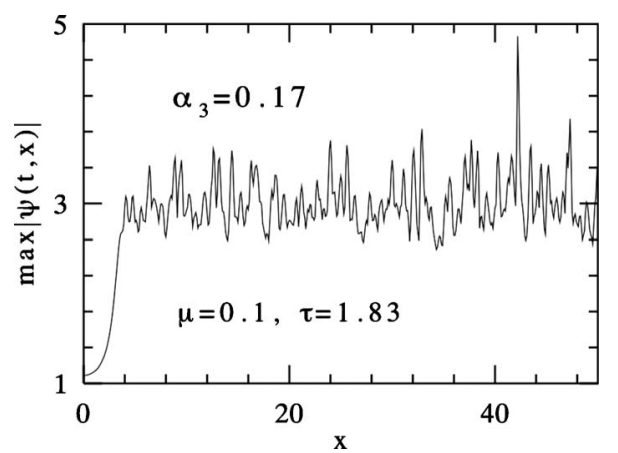

FIG. 6. Numerical simulations for the Hirota equation, showing the field maxima on evolution. Parameters of the simulation are presented inside of this figure.

\section{ROGUE WAVES IN A RANDOM FIELD}

The rational solutions that we have found analytically are regular. They have no singularities, contrarily to other types of rational solutions $[34,35]$. This implies that they may have a physical meaning. Do they have it in reality? In our previous work [20] we found that rational solutions of the NLSE equation are closely related to the rogue waves. They do appear in a CW field modulated by a chaotic perturbation.

In order to reveal the usefulness of our solutions, we made numerical simulations of the Hirota equation that start with a CW solution which is perturbed by a random function characterized by two parameters: the amplitude of the perturbation and its correlation length. The latter also defines the range of frequencies involved in the initial dynamics. Normally, we should fill the interval of frequencies that are modulationally unstable. Frequencies that are beyond that range may not grow directly due to the instability but they do grow due to the four-wave mixing process. Thus, the presence of these frequencies in the initial conditions is also important in the overall dynamics.

Specifically, when solving the Hirota equation we used the following initial conditions:

$$
\psi(0, t)=1+\mu a(t)
$$

where $a(t)$ is a complex stochastic function. Its real and imaginary parts are independent, and each has normal statistics with variance unity and a Gaussian correlation function. $\mu$ renormalizes the variance of the whole initial condition to the desired (small) value. We take $\mu=0.1$ in the simulations presented below. It is essential that the real and imaginary parts of the function $a(t)$ are independent. We know that the amplitude of a purely periodic perturbation has to have a specific complex value to lead to a homoclinic orbit (see Fig. 1 of [14]). Any other complex number results in an orbit periodic in the $x$ variable. Thus, the randomness of the initial condition would not be complete without this requirement.

Starting from the above initial condition leads to the exponential growth of those spectral components which are modulationally unstable. Of course, the initial amplitudes of these components are different for each realization. The numerical results for a particular choice of $\alpha, \mu=0.1, \tau$, and a particular realization are shown in Fig. 6. Here, for each $x$ we 
plot the maximum value of the field amplitude found in the whole range of the $t$ coordinate. In our simulations, the $t$ interval is taken to be $[-300,300]$ which is sampled with 65536 points. This result corresponds to a special realization, as usually the absolute maximum does not reach such a high value as 5 .

The initial growth in Fig. 6 is almost perfectly exponential and related to a particular maximum emerging from the otherwise chaotic field. The growth continues until $x$ is around 4. Further evolution becomes chaotic in the $x$ direction with the abrupt jumps in the curve due to the fact that the position of the absolute maximum changes its temporal position as the numerical grid is very wide and therefore comprises a big number of local maxima at each $x$. The general evolution of the two-dimensional field $\psi$ is somewhat different from the case of the NLSE [19]. However, these detailed features will be discussed elsewhere. What is important for us here is the field profile around the absolute maximum of the curve found in Fig. 6. Namely, we are interested in the point which is close to $x=42.2$. Once we find the value $t$ where this maximum occurs, we can plot the color contour plot of the transverse profile of the field in Fig. 7. This plot is very similar to that presented in Fig. 5 for the exact rational solution of the second order. If we eliminate the radiation waves around the peak this would be the rational solution of the second order with high accuracy. The amplitude at the point of maximum in the latter case is close to 5 which is another confirmation that we are dealing with the rational solution of the second order. Thus, the rational solutions of the second order can play the role of the rogue waves in the field described by the Hirota equation. Our numerical simulations confirm this.

\section{CONCLUSIONS}

In conclusion, we have modified the Darboux transformation method to find rational solutions of the Hirota equation

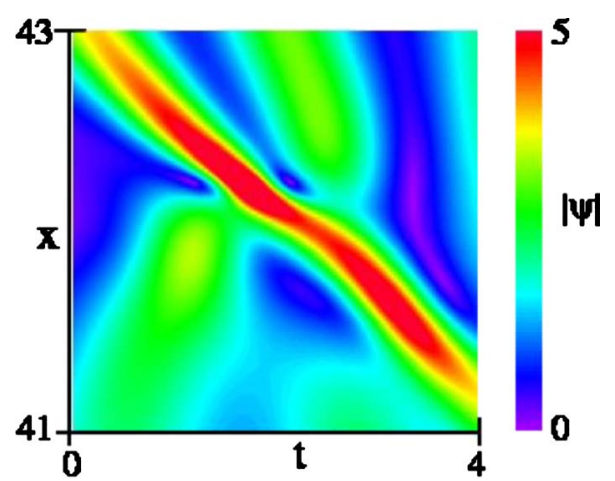

FIG. 7. (Color online) Numerical solution of the Hirota equation around a maximum of the field which approximately equals to 5 , for $\alpha_{3}=0.17$ (absolute maximum in Fig. 6). Note its similarity to the rogue wave shown in Fig. 5.

of the first and second orders. Our numerical simulations have shown that the second-order rational solution is a prototype of a rogue wave produced by the Hirota equation from random initial conditions with a given small amplitude of chaotic perturbations. Given that Hirota equation has the terms which may describe more accurately the waves in the ocean and in optical fibers than the nonlinear Schrödinger equation, our findings could be important for further progress in understanding the rogue wave phenomenon.

\section{ACKNOWLEDGMENTS}

The authors acknowledge the support of the Australian Research Council (Discovery Project No. DP0663216). J.M.S.C. acknowledges support from the Spanish Ministerio de Ciencia e Innovación under contract Nos. FIS2006-03376 and FIS2009-09895.
[1] C. Garrett and J. Gemmrich, Phys. Today 62 (6), 62 (2009).

[2] M. Onorato, T. Waseda, A. Toffoli, L. Cavaleri, O. Gramstad, P. A. E. M. Janssen, T. Kinoshita, J. Monbaliu, N. Mori, A. R. Osborne, M. Serio, C. T. Stansberg, H. Tamura, and K. Trulsen, Phys. Rev. Lett. 102, 114502 (2009).

[3] J. M. Dudley, G. Genty, F. Dias, B. Kibler, and N. Akhmediev, Opt. Express 17, 21497 (2009).

[4] T. B. Benjamin and J. E. Feir, J. Fluid Mech. 27, 417 (1967).

[5] V. I. Bespalov and V. I. Talanov, Zh. Eksp. Teor. Fiz. Pis'ma Red. 3, 471 (1966) [JETP Lett. 3, 307 (1966)].

[6] V. E. Zakharov and L. A. Ostrovsky, Physica D 238, 540 (2009).

[7] E. Fermi, J. Pasta, and S. Ulam, Los Alamos Report No. LA1940, 1955 (unpublished); E. Fermi, Collected Papers, edited by E. Segré (University Chicago Press, Chicago, 1965), Vol II, p. 978.

[8] N. Akhmediev, Nature (London) 413, 267 (2001).

[9] K. B. Dysthe and K. Trulsen, Phys. Scr. T82, 48 (1999).

[10] D. Clamond, M. Francius, J. Grue, and C. Kharif, Eur. J.
Mech. B/Fluids 25, 536 (2006).

[11] I. Ten and H. Tomita, Chikushi Campus, Kyushu University Reports of RIAM Symposium No. 17SP1-2, Kasuga, Fukuoka, Japan, 10-11 March 2006 (unpublished).

[12] V. V. Voronovich, V. I. Shrira, and G. Thomas, J. Fluid Mech. 604, 263 (2008).

[13] V. I. Shrira and V. V. Geogjaev, J. Eng. Math. (2009).

[14] N. Akhmediev and V. I. Korneev, Teor. Mat. Fiz. 69, 189 (1986) [Theor. Math. Phys. 69, 1089 (1986)].

[15] N. Akhmediev, A. Ankiewicz, and M. Taki, Phys. Lett. A 373, 675 (2009).

[16] D. H. Peregrine, J. Aust. Math. Soc. Ser. B, Appl. Math. 25, 16 (1983).

[17] N. Akhmediev, V. M. Eleonskii, and N. E. Kulagin, Teor. Mat. Fiz. 72, 183 (1987) [Theor. Math. Phys. 72, 809 (1987)].

[18] N. Akhmediev, A. Ankiewicz, and J. M. Soto-Crespo, Phys. Rev. E 80, 026601 (2009).

[19] N. Akhmediev, J. M. Soto-Crespo, and A. Ankiewicz, Phys. Lett. A 373, 2137 (2009). 
[20] N. Akhmediev, A. Ankiewicz, and J. M. Soto-Crespo, Phys. Rev. A 80, 043818 (2009).

[21] Yu. V. Sedletsky, Zh. Eksp. Teor. Fiz. 124, 200 (2003) [J. Exp. Theor. Phys. 97, 180 (2003)].

[22] A. V. Slunyaev, Zh. Eksp. Teor. Fiz. 128, 1061 (2005) [J. Exp. Theor. Phys. 101, 926 (2005)].

[23] A. Ankiewicz, N. Devine, and N. Akhmediev, Phys. Lett. A 373, 3997 (2009).

[24] A. R. Osborne, Nonlinear Processes Geophys. 4, 29 (1997).

[25] R. Hirota, J. Math. Phys. 14, 805 (1973).

[26] M. Lakshmanan and S. Ganesan, J. Phys. Soc. Jpn. 52, 4031 (1983).

[27] N. Akhmediev, V. I. Korneev, and N. V. Mitskevich, Izv. Vyssh. Uchebn. Zaved., Radiofiz. 33, 95 (1990) [Radiofiz. 33,
111 (1990)]

[28] G. L. Lamb, Elements of Soliton Theory (Wiley, New York, 1980).

[29] R. Grimshaw et al., Stud. Appl. Math. 114, 189 (2005).

[30] N. Akhmediev and A. Ankiewicz, Soltions, Nonlinear Pulses and Beams (Chapman \& Hall, London, 1997).

[31] G. Van Simaeys, Ph. Emplit, and M. Haelterman, Phys. Rev. Lett. 87, 033902 (2001).

[32] A. Mussot, E. Louvergneaux, N. Akhmediev, F. Reynaud, L. Delage, and M. Taki, Phys. Rev. Lett. 101, 113904 (2008).

[33] V. B. Matveev and M. A. Salle, Darboux Transformations and Solitons (Springer, Berlin-Heidelberg, 1991).

[34] A. N. W. Hone, J. Phys. A 30, 7473 (1997).

[35] P. A. Clarkson, Eur. J. Appl. Math. 17, 293 (2006). 